\title{
Limits of Convolution Sequences of Measures on a Compact Topological Semigroup
}

\author{
M. ROSENBLATT
}

Introduction. Limit properties of the convolution sequence of a regular measure on a compact topological semigroup are examined in this paper. Similar questions, as they arise in the case of a compact group, were examined by KAWADA \& Ito [4]. Recently Bellman [1] and Grenander [3] considered special limit theorems for products of independent identically distributed random operators. Such problems are closely related to those in this paper. It should be noted that similar questions arise when considering the structure of stationary stochastic processes [7]. Various results on compact semigroups are used in characterizing the class of limit measures [5], [6], [8].

Basic Concepts. Let $S$ be a compact Hausdorff space. Take B as the Borel field generated by the open sets of the topology. Further, let $S$ be a topological semigroup. This means that there is a product for pairs of elements of $S$ and that the product operation is continuous with respect to the given topology (see [5], [6]). Let $B \times B$ be the Borel field in the product space $S \times S$ generated by the Borel field $B$ in the component spaces. The set

$$
A_{B}=\{(x, y) \mid x y \varepsilon B\} \varepsilon B \times B
$$

if $B \varepsilon \&$ since it is true for open sets $B$. We can define the product measure $\nu \times \mu$ on $\beta \times B$ in the usual manner [2] and the convolution $\nu * \mu$ of two measures as

$$
\nu^{*} \mu(B)=\int c_{B}(x y) d(\nu \times \mu)((x, y))
$$

for $B \varepsilon \beta$, where $c_{B}$ is the characteristic function of $B$.

In Lemmas 1 and 2 some simple properties of a measure on a topological space are obtained. No use is made of the semigroup property.

Lemma 1. Let $\nu$ be a measure on a Borel field $\mathcal{G}$ of a topological space. Let $\mathcal{F}$ be the collection of sets $E \varepsilon$ B such that for any $\epsilon>0$ there are open and closed sets

\footnotetext{
This research was supported by the Office of Naval Research under contract Nonr 908(10).
} Reproduction in whole or in part is permitted for any purpose of the United States Government. 
$O, C$ such that

$$
C \subset E \subset O
$$

and

$$
\nu(O-E)<\epsilon, \quad \nu(E-C)<\epsilon .
$$

Then $\mathfrak{F}$ is a monotone field.

Consider any two sets $E_{1}, E_{2} \varepsilon$ F. Given any $\epsilon>0$ there are open sets $O_{i}$ and closed sets $C_{i}$,

$$
C_{i} \subset E_{i} \subset O_{i}, \quad i=1,2,
$$

with

$$
\nu\left(O_{i}-E_{i}\right)<\frac{1}{2} \epsilon, \quad \nu\left(E_{i}-C_{i}\right)<\frac{1}{2} \epsilon
$$

But

$$
\begin{aligned}
& \nu\left(O_{1} \cup O_{2}-E_{1} \cup E_{2}\right) \leqq \nu\left(\left(O_{1}-E_{1}\right) \cup\left(O_{2}-E_{2}\right)\right)<\epsilon, \\
& \nu\left(E_{1} \cup E_{2}-C_{1} \cup C_{2}\right) \leqq \nu\left(\left(E_{1}-C_{1}\right) \cup\left(E_{2}-C_{2}\right)\right)<\epsilon .
\end{aligned}
$$

Thus $E_{1} \cup E_{2} \varepsilon F$.

We now show that $E_{1}-E_{2} \varepsilon$ F. There is an open set $O_{1,2}$ and a closed set $C_{2}$ such that

$$
E_{1} \cup E_{2} \subset O_{1,2}, \quad C_{2} \subset E_{2}
$$

with

$$
\nu\left(O_{1,2}-E_{1} \cup E_{2}\right)<\frac{1}{2} \epsilon, \quad \nu\left(E_{2}-C_{2}\right)<\frac{1}{2} \epsilon .
$$

Now $O_{1,2}-C_{2}$ is open and $E_{1}-E_{2} \subset O_{1,2}-C_{2}$. Further,

$$
O_{1,2}-C_{2}-\left(E_{1}-E_{2}\right)=\left(O_{1,2}-E_{1} \cup E_{2}\right) \cup\left(E_{2}-C_{2}\right)
$$

and hence

$$
\nu\left(O_{1,2}-C_{2}-\left(E_{1}-E_{2}\right)\right)<\epsilon .
$$

Note that the empty set $\varnothing$ and the whole space $\Omega$ obviously belong to $F$. One can reduce the problem of finding an approximating closed set within $E_{1}-E_{2}$ to the argument given above by complementation. Thus $E_{1}-E_{2} \varepsilon \mathcal{F}$.

Now consider a monotone sequence of sets $E_{n} \varepsilon F, E_{n} \uparrow E$. We shall show that $E \varepsilon$ F. Let $n$ be so large that $\nu\left(E-E_{n}\right)<\frac{1}{2} \epsilon$. There is then a closed set $C \subset E_{n}$ with $\nu\left(E_{n}-C\right)<\frac{1}{2} \epsilon$. But then $\nu(E-C)<\epsilon$. Given each $E_{n}$ there is an open set $O_{n}, E_{n} \subset O_{n}$, with $\nu\left(O_{n}-E_{n}\right)<\epsilon / 2^{n+1}$. Now $O=\cup O_{n}$ is open, $E \subset \cup O_{n}$, and

$$
\nu(O-E) \leqq \nu\left(\cup\left(O_{n}-E_{n}\right)\right)<\epsilon .
$$

Thus $\mathfrak{F}$ is a monotone field. 
If $\nu$ is a measure on the Borel field $g$ and the monotone field $\mathcal{F}$ of Lemma 1 is identical with $G$, we say that $\nu$ is regular on $G$.

Lemma 2. If $\nu, \mu$ are regular measures on $B$, the product measure $\nu \times \mu$ is regular on $B \times B$. If the product measure $\nu \times \mu$ is regular, the factor measures $\nu, \mu$ are regular.

Clearly the product sets with projections on the factor spaces elements of $B$ have the property of Lemma 1 . Therefore the $\sigma$-field $\beta \times B$ generated by these sets has this property.

Conversely let $\nu \times \mu$ be regular and $B \varepsilon$ B. Then there is a closed set $C \varepsilon$ $B \times B, C \subset B \times \Omega$ with $\nu \times \mu(B \times \Omega-C)<\epsilon$. Let $C^{\prime} \varepsilon \beta \times B$ be the set of all points whose $x$ projection is the same as the $x$ projection of some point of $C$. The set $C$ is closed since $f((x, y))=x$ is continuous. Let the $x$ projection of the set $C^{\prime}$ be $C^{\prime \prime} \varepsilon$ B. Then $C^{\prime \prime} \subset B$ and

$$
\nu\left(B-C^{\prime \prime}\right)=\nu \times \mu\left(B \times \Omega-C^{\prime}\right) \leqq \nu \times \mu(B \times \Omega-C) .
$$

Lemma 3. If $\nu, \mu$ are regular measures on $B$, the convolution $\nu * \mu$ is regular (on $B$ ).

Let $B \varepsilon$ B. Set

$$
A_{B}=\{(x, y) \mid x y \varepsilon B\} .
$$

Now $A_{B} \varepsilon B \times B$, and hence by the regularity of $\nu \times \mu$ there is a closed set $C^{\prime} \subset A_{B}$ with

$$
\nu \times \mu\left(A_{B}-C^{\prime}\right)<\epsilon \text {. }
$$

Let

$$
C=\left\{z \mid z=x y \text { for some pair }(x, y) \varepsilon C^{\prime}\right\} .
$$

It is clear that $C^{\prime} \subset A_{C} \subset A_{B}$. Thus

$$
\nu * \mu(B-C)=\nu \times \mu\left(A_{B}-A_{C}\right)<\epsilon .
$$

Further, $C$ is a closed set since it is the image of a continuous function on the closed set $C^{\prime}$. We can find an open set containing $B$ with the desired properties by complementation.

Let us now consider the family of regular probability measures $\mathfrak{N}$ on the compact topological semigroup $S$. We shall say that the sequence of regular probability measures $\mu_{n}$ on $S$ converges to a regular probability measure $\mu, \mu_{n} \rightarrow \mu$, if

$$
\int f d \mu_{n} \rightarrow \int f d \mu
$$

for every continuous function $f$ on $S$.

Since the continuous functions on a compact space are bounded, it is clear that it is enough to have convergence for continuous functions $f, 0 \leqq f \leqq 1$. 
Let $I$ be the closed unit interval $[0,1]$. Consider the product space $\Pi_{f} I_{f}$ where the coordinate spaces $I_{f}$ are indexed by continuous functions $f, 0 \leqq f<1$, with the usual Tychonov topology [2]. $\prod I_{f}$ is a compact space since $I_{f}$ is compact. Each measure $\mu_{\varepsilon} \mathfrak{I}$ can be considered as a point in $\prod I_{f}$ with component in the $f^{t h}$ coordinate space $I_{f}=\int f d \mu$. Let us consider a limit point $c \varepsilon \prod I_{f}$ of the family of measures $\mathfrak{T}$. Clearly $c_{f} \geqq 0$ for all $f$ and $c_{1}=1$ ( 1 is the function $f \equiv 1)$. Further, given any two functions $f_{1}, f_{2}, 0 \leqq f_{1}, f_{2}, f_{1}+f_{2} \leqq 1$, and any $\epsilon>0$ there is a measure $\mu \varepsilon \mathfrak{T}$ in the neighborhood

$$
\left\{c^{\prime}|| c_{f_{3}}^{\prime}-c_{f_{1}}|<\epsilon,| c_{f_{2}}^{\prime}-c_{f_{2}}|<\epsilon,| c_{f_{1}+f_{2}}^{\prime}-c_{f_{1}+f_{2}} \mid<\epsilon\right\} .
$$

But this implies that $c_{f_{1}+f_{2}}=c_{f_{1}}+c_{f_{2}}$. Let $c_{f}=L(f) . L(f)$ is a functional on the continuous functions $f, 0 \leqq f \leqq 1$. We can similarly show that $c_{\alpha f}=\alpha c_{f}$ for $\alpha \geqq 0$ if $0 \leqq \alpha f, f \leqq 1$. Further, $\left|c_{f}\right| \leqq\|f\|$ if $0 \leqq f \leqq 1$ (absolute supremum norm). We now extend $L$ to all continuous $f$. First suppose $f \geqq 0$. Then there is a positive constant $\alpha$ such that $0 \leqq \alpha f \leqq 1$. Let $L(f)=L(\alpha f) / \alpha$. Thus $L(f)$ is defined on all continuous nonnegative $f$. If $f$ is not nonnegative, introduce $f^{+}=\max (0, f)$ and $f^{-}=f-f^{+}$. Then $f^{+},-f^{-}$are nonnegative continuous functions. Let $L(f)=L\left(f^{+}\right)-L\left(-f^{-}\right) . L$ is linear on the continuous functions since $L(\alpha f)=\alpha L(f), L\left(f_{1}+f_{2}\right)=L\left(f_{1}\right)+L\left(f_{2}\right) . L$ is positive, that is, $L(f) \geqq 0$ if $f \geqq 0$. Further, $L(f) \leqq\|f\|$ for $f \geqq 0$. But then

$$
\begin{aligned}
|L(f)|=\left|L\left(f^{+}\right)-L\left(-f^{-}\right)\right| & \leqq \max \left(L\left(f^{+}\right), L\left(-f^{-}\right)\right) \\
& \leqq \max \left(\left\|f^{+}\right\|,\left\|-f^{-}\right\|\right)=\|f\|
\end{aligned}
$$

for all continuous $f$, and thus $\|L\| \leqq 1$. By the Riesz representation theorem [2] there is a regular probability measure $\mu$ on $S$ (since $L(1)=1$ ) such that $L(f)=$ $\int f d \mu$. Therefore $c \varepsilon \mathfrak{T}$ and $\mathfrak{T}$, as a point set in $\Pi I_{f}$, is closed. Thus $\mathfrak{T}$ is compact.

Lemma 4. The set of regular probability measures $\operatorname{Tr}$ on $S$ is a compact set in $\prod I_{f}$.

Now let us investigate the continuity of the convolution operation on the set of regular probability measures. First consider a continuous function $f(x, y)$ on $S \times S$. It is easy to see that the set of finite sums of products of continuous functions of the form

$$
\sum_{i=1}^{N} g_{i}(x) h_{i}(y)
$$

with $g_{i}(x), h_{i}(y)$ continuous on $S$ is an algebra of functions that separate points. For if $(x, y) \neq\left(x^{\prime}, y^{\prime}\right)$ either $x \neq x^{\prime}$ or $y \neq y^{\prime}$. Suppose $x \neq x^{\prime}$. Take a continuous function $g(x)$ that separates $x, x^{\prime}$ and let $h(y) \equiv 1$. Then $g(x) h(y)=g(x)$ separates $(x, y),\left(x^{\prime}, y^{\prime}\right)$. Since $S \times S$ is compact, by the Stone-Weierstrass theorem any continuous function $f(x, y)$ on $S \times S$ can be uniformly approximated by a function of the form (20) with $g_{i}, h_{i}$ continuous on $S$. We shall now prove Lemma 5. 
Lemma 5. The convolution operation is continuous on the probability measures Tr.

It will be enough to show that the inverse image of a set in the subbasis for regular probability measures is open. Let us consider two probability measures $\nu, \mu \varepsilon \mathfrak{M}$. A subbasis neighborhood of $\nu * \mu$ is of the form

$$
\left\{c|| \int f d \nu * \mu-c \mid<\epsilon\right\}
$$

where $f$ is a continuous function, $0 \leqq f \leqq 1$. Now

$$
\int f(x) d \nu * \mu(x)=\int f(x y) d \nu \times \mu((x, y)) .
$$

But $f$ is a continuous function of $(x, y)$, and hence there is an approximating function

$$
u(x, y)=\sum_{i=1}^{N} g_{i}(x) h_{i}(y)
$$

with $h_{i}, g_{i}$ continuous on $S$ such that

$$
|u(x, y)-f(x, y)|<\frac{1}{4} \epsilon .
$$

Then

$$
\left|\int[u(x, y)-f(x y)] d \nu \times \mu((x, y))\right|<\frac{1}{4} \epsilon .
$$

Let $M<\infty$ be such that $\left|g_{i}\right|,\left|h_{i}\right|<M$ for all $i$. Consider the following neighborhoods of $\nu, \mu$ respectively:

$$
\begin{aligned}
& N(\nu)=\left\{\eta|| \int g_{i}(x) d \eta(x)-\int g_{i}(x) d \nu(x) \mid<\delta ; i=1, \cdots, N\right\}, \\
& N(\mu)=\left\{\eta|| \int h_{i}(x) d \eta(x)-\int h_{i}(x) d \mu(x) \mid<\delta ; i=1, \cdots, N\right\} .
\end{aligned}
$$

If $\nu^{\prime} \varepsilon N(\nu), \mu^{\prime} \varepsilon N(\mu)$ then

$$
\begin{aligned}
& \left|\int u(x, y) d \nu \times \mu((x, y))-\int u(x, y) d \nu^{\prime} \times \mu^{\prime}((x, y))\right| \\
& \quad \leqq \sum_{i=1}^{N}\left|\int g_{i}(x) h_{i}(y)\left[d \nu \times \mu((x, y))-d \nu^{\prime} \times \mu^{\prime}((x, y))\right]\right| \leqq 2 N \delta M,
\end{aligned}
$$

and thus if $\delta<\epsilon / 4 N M$,

$$
\left|\int f d \nu * \mu-\int f d \nu^{\prime} * \mu^{\prime}\right|<\epsilon .
$$

The following theorem is an immediate consequence of the results obtained thus far. 
Theorem 1. The family of regular probability measures $\mathfrak{T}$ on $S$ with the convolution operation as a multiplicative operation is a compact Hausdorff topological semigroup.

Limit Properties of Convolution Sequences. Let $\nu$ be a regular probability measure on $S$. We shall now consider limit properties of the sequence of measures $\nu^{(1)}=\nu, \nu^{(2)}=\nu * \nu, \cdots, \nu^{(n+1)}=\nu^{(n)} * \nu, \cdots$ generated from $\nu$ by convolution. A classical probability problem of this sort arises in considering the limit properties of sums of independent, identically distributed random variables. The values taken on by the random variables (since they are numerical-valued) commute with each other. In our case, we are interested in limit properties of products of independent, identically distributed elements of a semigroup (possibly a semigroup of operators) and a non-abelian semigroup is of considerable interest to us.

The notion of the spectrum of a regular measure $\nu$ on $S$ is of particular interest. The spectrum $\Sigma(\nu)$ of a measure $\nu \varepsilon \mathfrak{I}$ consists of the points $s \varepsilon S$ such that any open set $O$ containing $s$ has positive $\nu$ measure. The spectrum is clearly a closed set. Given any two subsets $A, B$ of the semigroup $S$ let $A B=\left\{s s^{\prime} \mid s \varepsilon A, s^{\prime} \varepsilon B\right\}$. Now the closure of the set of elements $\bigcup_{n}(\Sigma(\nu))^{n}$ is a closed semigroup and hence is compact. This is the relevant semigroup insofar as the sequence of convolutions $\nu^{(n)}$ is concerned. Let us call this subsemigroup the semigroup generated by the spectrum of $\nu$. Since this semigroup is of importance in considering the limit properties of the sequence $\nu^{(n)}$, we shall take $S=\overline{\mathcal{U}_{n}(\Sigma(\nu))^{n}}$ ( $\bar{A}$ is the closure of $A$ ).

An element $e$ of $S$ is called an idempotent if $e^{2}=e$. Every compact Hausdorff semigroup has at least one idempotent [5]. By a left (right) ideal of $S$ we mean a nonvacuous subset $L(R)$ of $S$ such that $S L \subset L(R S \subset R)$. If $M$ is both a left and right ideal of $S, M$ is called a (two-sided) ideal of $S$. The minimal twosided ideal $K$ of $S$ exists and is given by $K=\bigcap_{\text {. SeS [5]. The minimal ideal } K}$ is called the kernel of the semigroup $S$. $K$ is closed and hence compact [5].

We shall say that $\mu$ is a limit measure of the sequence $\nu^{(n)}, n=1,2, \cdots$, if there is a subsequence $n_{k}$ such that

$$
\mu=\lim _{k \rightarrow \infty} \nu^{\left(n_{k}\right)} \text {. }
$$

Theorem 2. Let $\nu$ be a regular probability measure on the compact Hausdorff semigroup S. Every limit measure $\mu$ of the sequence $\nu^{(n)}$ ( $\nu$ regular) has all its mass concentrated on the kernel of the semigroup generated by the spectrum of $\nu$.

It is clear that we can take $S=\overline{\bigcup(\Sigma(\nu))^{n}}$. Let $k$ be an element of the kernel $K$ of the semigroup generated by the spectrum of $\nu$. Let $N(k)$ be any neighborhood of $k$. Then for $m$ sufficiently large there are elements $s_{1}, \cdots, s_{m} \varepsilon \Sigma(\nu)$ such that $\prod_{i=1}^{m} s_{i} \varepsilon N(k)$. By the continuity of the multiplicative operation there are neighborhoods $N\left(s_{i}\right)$ of $s_{i}, i=1, \cdots, m$, such that $\prod_{i=1}^{m} N\left(s_{i}\right) \subset N(k)$. Let $O$ be any open set containing $K$. Then there must be a neighborhood of $k, N(k)$, 
such that $S N(k), N(k) S \subset O$. For given any point $s \varepsilon S$ there are neighborhoods $N_{1 s}(k), N_{2 s}(k)$ of $k$ and a neighborhood $N(s)$ of $s$ such that $N_{1 s}(k) N(s)$, $N(s) N_{2 s}(k) \subset O$ since $S K=K S=K$. Let $N_{s}(k)=N_{1 s}(k) \cap N_{2 s}(k)$. Then $N(s) N_{s}(k), N_{s}(k) N(s) \subset O$. The open sets $N(s)$ cover $S$, and hence there are a finite number of $s_{i}^{1}$ 's, $i=1, \cdots, n$, such that the $N\left(s_{i}^{1}\right)$ cover $S$ (because of the compactness of $S) . N(k)=\bigcap_{1}^{n} N_{s^{2}}(k)$ is a neighborhood of $k$ such that $S N(k), N(k) S \subset O$. Given this neighborhood $N(k)$ of $k$ choose a finite number of elements $s_{1}, \cdots, s_{m}$ with corresponding neighborhoods $N\left(s_{i}\right), i=1, \cdots, m$, such that $\prod_{i=1}^{m} N\left(s_{i}\right) \subset N(k)$. Now consider the infinite product space of sequences $\left(t_{1}, t_{2}, \cdots\right)$ of elements $t_{i}$ of $S$ with product measure generated by $\nu$. Now $\nu\left(N\left(s_{i}\right)\right)>0, i=1, \cdots, m$. By the lemma of Borel-Cantelli there is at least one block of $m$ successive elements from $N\left(s_{1}\right), \cdots, N\left(s_{m}\right)$ respectively in any such sequence with measure one. Take $n$ sufficiently large so that the measure of the set with a block of $m$ such successive elements in truncated sequences of length $n$ is greater than $1-\epsilon$. It then follows that $\nu^{(n+1)}(O)>1-\epsilon$. But this implies that for any limit measure $\mu$ of the sequence $\nu^{(n)}, \mu(O)=1$. However, this is true for any open set $O$ containing $K$. Therefore $\mu(K)=1$.

Lemma 6. Let $f((x, y))$ be a continuous function on $S \times S$ where $S$ is a compact Hausdorff space. Then the family of functions $f_{y}(x)=f((x, y))$ defined on $S$ and indexed by $y \in S$ is equicontinuous for all $y \varepsilon S$.

To say that the family $\left\{f_{y}(x)\right\}$ is equicontinuous is equivalent to stating that for any given $\epsilon>0$ and any $x \varepsilon S$ there is a neighborhood $N(x)$ of $x$ such that for all $x^{\prime} \varepsilon N(x)$

$$
\left|f_{y}\left(x^{\prime}\right)-f_{y}(x)\right|<\epsilon
$$

independently of $y$. Suppose the family is not equicontinuous. Then for some $\epsilon>0$ and some $x \varepsilon S$ there is no neighborhood $N(x)$ of $x$ such that $\left|f_{y}\left(x^{\prime}\right)-f_{y}(x)\right|<\epsilon$ for all $x^{\prime} \varepsilon N(x)$ and all $y$. Index the neighborhoods $N_{\alpha}$ of $x$ by $\alpha$. Given any neighborhood $N_{\alpha}$ of $x$ there is a point $\left(x_{\alpha}, y_{\alpha}\right) \varepsilon S \times S$, $x_{\alpha} \varepsilon N_{\alpha}$, such that $\left|f_{y_{\alpha}}\left(x_{\alpha}\right)-f_{y_{\alpha}}(x)\right| \geqq \epsilon$. The indices $\alpha$ are partially ordered if we set $\alpha \leqq \alpha^{\prime}$ when $N_{\alpha}, \subset N_{\alpha}$. Further, the indices are a directed set since any finite subset of them has an upper bound. Thus $\left\{x_{\alpha}\right\}$ and $\left\{\left(x_{\alpha}, y_{\alpha}\right)\right\}$ are generalized sequences of elements in $S$ and $S \times S$ respectively [2]. Further, $\lim _{\alpha} x_{\alpha}=x$. The generalized sequence $\left(x_{\alpha}, y_{\alpha}\right)$ has a cluster (limit) point $(x, y)$. But then $\left|f_{y}(x)-f_{y}(x)\right|=|0|=0 \geqq \epsilon>0$, a contradiction.

Theorem 3. Let $\nu$ be a regular probability measure on the compact Hausdorff semigroup $S$. There is then a unique limit measure of the averaged convolution sequence

Further,

$$
\mu=\lim _{n} \frac{1}{n} \sum_{j=1}^{n} \nu^{(j)} .
$$

$$
\mu * \nu=\nu * \mu=\mu^{(2)}=\mu .
$$


Consider the regular probability measure $\nu$ as an operator $T$ on the family of continuous functions $f$ on $S$, that is,

$$
(T f)(x)=\int f(x y) d \nu(y) .
$$

The family of functions $f_{y}(x)=f(x y)$ is an equicontinuous family of functions by Lemma 6 , and hence $T$ maps the continuous functions into continuous functions. Consider iterates of the operator $T, T^{n}$, acting on $f$. Then

$$
\left(T^{n} f\right)(x)=\int\left(T^{n-1} f\right)(x y) d \nu(y)=\int f(x y) d \nu^{(n)}(y) .
$$

Let

$$
\bar{T}^{n}=\frac{1}{n} \sum_{i=1}^{n} T^{i} .
$$

The sequence of functions $\left(T^{n} f\right)(x)$ is an equicontinuous family of functions since $f_{y}(x)=f(x y)$ is equicontinuous. This implies that $\left(\bar{T}^{n} f\right)(x)$ is equicontinuous and hence $\left\{\bar{T}^{n} f\right\}$ is strongly compact (sequentially). Further, $\|T\|=1$ and $T^{n} f / n \rightarrow 0$. Therefore $\bar{T}^{n} \rightarrow \bar{T}$ strongly where $\bar{T}$ is linear,

$$
\|\bar{T}\|=1, \quad \bar{T} T=T \bar{T}=\bar{T}^{2}=\bar{T},
$$

and $\bar{T}$ is positivity preserving. Hence there is a regular probability measure $\mu$ such that

$$
\int \bar{T} f(x) d \nu(x)=\int f(x) d \mu(x) .
$$

But then

$$
\frac{1}{n} \int f(x) d\left(\sum_{j=1}^{n} \nu^{(i)}(x)\right) \rightarrow \int f(x) d \mu(x)
$$

for all continuous $f$, and hence $(1 / n) \sum_{j=1}^{n} \nu^{(i)} \rightarrow \mu$.

Limit Measures on the Kernel of the Semigroup. Consider limit measures of the kind discussed in Theorem 3. These measures are the idempotents in the semigroup of probability measures on $S$. For simplicity, we restrict ourselves to those measures $\nu$ in this semigroup of measures whose spectrum generates $S$. By Theorem 2 the limit measure $\mu$ of $(1 / n) \sum_{1}^{n} \nu^{(i)}$ is concentrated on the kernel $K$ of $S$. In Theorem 4 we shall show that the spectrum of a limit measure $\mu$ is $K$. The detailed structure of such measures will be examined in this section.

However, we will require certain basic results on the structure of the kernel $K$ of a compact Hausdorff semigroup. When studying $K$, it is natural to look at it with the relative topology. $K$ is a completely simple semigroup, that is, $K k K=K$ for all $k \varepsilon K$ and $K$ has a minimal left and a minimal right ideal [8]. Every compact completely simple semigroup can be represented as the product 
space $T \times X \times Y$ of a compact topological group $T$ and compact Hausdorff spaces $X$ and $Y$ where the multiplication is given by

$$
(t, x, y)\left(t^{\prime}, x^{\prime}, y^{\prime}\right)=\left(t \varphi\left(x, y^{\prime}\right) t^{\prime}, x^{\prime}, y\right)
$$

with $\varphi$ a continuous function on the product space $X \times Y$ into $T$ [8]. We shall therefore identify $K$ with such a space $T \times X \times Y$ and the accompanying $\varphi$ function.

Theorem 4. Let $S$ be a compact Hausdorff semigroup and $\nu$ a regular measure on $S$ whose spectrum generates $S$. The spectrum of the limit measure $\mu=\lim (1 / n)$ $\sum_{1}^{n} \nu^{(i)}$ is $K$, the kernel of $S$.

Let $k$ be any element of $K$. Let $N(k)$ be a neighborhood of $k$. It is enough to show that $\mu(N(k))>0$. Any point $k \varepsilon K$ has the form $k=(t, x, y)$. Let $x=x(k)$, $y=y(k)$ be the $x, y$ coordinates of $k$. Further, let $N_{x, y}(k)$ be the collection of all points $k$ (in $K$, of course) with $x, y$ coordinates the same as those of points in $N(k) \cap K$. Take $k^{\prime}$ to be an element in the spectrum of $\mu$. There is then a neighborhood $N_{1}(k)$ such that

$$
N_{1}(k) k^{\prime} N_{1}(k) \subset N_{x, y}(k) .
$$

This follows from the continuity of the multiplicative operation. But then $\mu\left(N_{x, y}(k)\right)>0$ for any neighborhood $N(k)$ of $k$. For

$$
\mu\left(N_{x, \nu}(k)\right)=\int c_{N_{x, y}(k)}\left(s_{1} s_{2} s_{3}\right) d \nu^{(n)}\left(s_{1}\right) d \mu\left(s_{2}\right) d \nu^{(n)}\left(s_{3}\right)
$$

and for some $n$

$$
\nu^{(n)}\left(N_{x, y}(k)\right)>0 .
$$

Thus there is a point $k^{\prime \prime} \varepsilon N_{x, y}(k)$ in the $\mu$ spectrum.

Given the neighborhood $N(k)$ there is a point $k^{\prime} \varepsilon N(k)$ with a corresponding point $k^{\prime \prime} \varepsilon N_{x, y}(k), x\left(k^{\prime \prime}\right)=x\left(k^{\prime}\right), y\left(k^{\prime \prime}\right)=y\left(k^{\prime}\right)$, that is, in the $\mu$ spectrum. There are then points $k_{1}^{\prime \prime}, k_{2}^{\prime \prime}$ such that $k_{1}^{\prime \prime} k^{\prime \prime} k_{2}^{\prime \prime}=k^{\prime}$. This implies that there are neighborhoods $N\left(k_{1}^{\prime \prime}\right), N\left(k^{\prime \prime}\right), N\left(k_{2}^{\prime \prime}\right)$ of $k_{1}^{\prime \prime}, k^{\prime \prime}, k_{2}^{\prime \prime}$ such that

$$
N\left(k_{1}^{\prime \prime}\right) N\left(k^{\prime \prime}\right) N\left(k_{2}^{\prime \prime}\right) \subset N\left(k^{\prime}\right) \subset N(k)
$$

by the continuity of the multiplicative operation and the structure of $K$. Then all of $K$ is the spectrum of $\mu$ since

$$
\nu^{(n)} * \mu * \nu^{(m)}=\mu .
$$

The proof is complete.

Let us now consider a product measure $\bar{\mu}$ on $K$ of the form

$$
\bar{\mu}=\chi \times \alpha \times \beta
$$

where $\chi$ is the normed Haar measure $(\chi(T)=1)$ of the compact topological group $T$ and $\alpha$ and $\beta$ are regular probability measures on $X, Y$ respectively. 
We shall show that such a measure is an idempotent on $K$. First consider a set $G \varepsilon B$ which is a product set, that is, $G=A \times B \times C$ where $A, B, C$ are sets of the Borel fields on $T, X, Y$ respectively. Let $s^{-1} G=\left\{s^{\prime} \mid s s^{\prime} \varepsilon G\right\}$. But

$$
\bar{\mu}^{(2)}(G)=\int \bar{\mu}\left(s^{-1} G\right) \bar{\mu}(d s)=\int_{\nu(s)_{e} C} \bar{\mu}\left(s^{-1} G\right) \bar{\mu}(d s) .
$$

Now

$$
\bar{\mu}\left(s^{-1} G\right)=\int_{B} \int_{Y} \chi\left(\left[t \varphi\left(x(s), y^{\prime \prime}\right)\right]^{-1} A\right) d \alpha\left(x^{\prime}\right) d \beta\left(y^{\prime \prime}\right)=\chi(A) \alpha(B)
$$

by the invariance of Haar measure. Thus

$$
\bar{\mu}^{(2)}(G)=\int_{y(s) \varepsilon C} \chi(A) \alpha(B) \bar{\mu}(d s)=\chi(A) \alpha(B) \beta(C)=\bar{\mu}(G) .
$$

Since $\bar{\mu}^{(2)}(G)=\bar{\mu}(G)$ for all product sets, the relation holds for all $G$ in the field of finite sums of product sets and hence for all $G$ in the Borel field $B$ generated by these sets.

Lemma 7. Let $K$ be a completely simple compact semigroup. Any measure $\bar{\mu}$ of the form (43) is an idempotent measure on $K$.

We shall now show that for a fairly wide class of completely simple compact semigroups, the $\bar{\mu}$ measures are the only idempotent measures.

Theorem 5. Let $K$ be a completely simple compact semigroup with the function $\varphi(x, y)$ of the form $\varphi(x, y)=\varphi_{1}(x) \varphi_{2}(y)$. Then every idempotent measure $\mu$ with spectrum the whole semigroup is a $\bar{\mu}$ measure.

Assume that the function $\varphi(x, y)=\varphi_{1}(x) \varphi_{2}(y)$. Introduce the following function $\psi$ of $k=(t, x, y)$ :

$$
\psi(k)=\varphi_{2}(y) t \varphi_{1}(x) .
$$

Now $\psi(k)$ is a continuous function of $k$ since $\varphi$ is. Suppose $\mu$ is an idempotent measure on $K$ with its spectrum $K$. Let $\nu$ be the derived measure

$$
\nu(A)=\mu(\psi(k) \varepsilon A)
$$

on the compact group $T$. Here $A$ is an element of the Borel field on $T$. Since $\mu$ is an idempotent measure on $K$, it follows that $\nu$ is an idempotent measure on $T$ [4]. The spectrum of $\nu$ is the whole group $T$ since the spectrum of $\mu$ is the whole semigroup $K$. However, this implies that $\nu$ is the normed Haar measure of $T$. Let

$$
\begin{aligned}
& \nu_{1}(A, C)=\mu\left(k \mid t \varphi_{1}(x) \varepsilon A, y \varepsilon C\right), \\
& \nu_{2}(A, B)=\mu\left(k \mid \varphi_{2}(y) t \varepsilon A, x \varepsilon B\right) .
\end{aligned}
$$


Now if $\mu$ is an idempotent measure $\mu^{(3)}=\mu$, and hence

$$
\nu(A \times B \times C)=\int \nu_{2}(d t, B) \nu\left(t^{\prime-1} A t\right) \nu_{1}\left(d t^{\prime}, C\right)=\nu_{2}(\cdot, B) \nu_{1}(\cdot, C) \nu(A)
$$

since $\nu$ is the Haar measure of $T$. Thus $\mu$ is a $\bar{\mu}$ measure.

Corollary. If $S$ is a compact Abelian Hausdorff semigroup, the kernel of $S$ is an Abelian group. Any idempotent measure is concentrated on the kernel. If the spectrum of the measure is the kernel, it is the normed Haar measure of the kernel.

This is almost an immediate consequence of Theorem 4.

We shall now show that the result of Theorem 5 is true for a general finite completely simple semigroup. This makes one suspect that Theorem 5 very likely holds without the restriction on $\varphi(x, y)=\varphi_{1}(x) \varphi_{2}(y)$.

Theorem 6. Let $K$ be a finite completely simple semigroup. Every idempotent measure $\mu$ with spectrum the whole semigroup is a $\bar{\mu}$ measure.

Since $\mu$ is idempotent

$$
\mu\left(k^{\prime}\right)=\sum_{k} \mu\left(k^{\prime} k^{-1}\right) \mu(k)
$$

where $k^{\prime} k^{-1}=\left\{a \mid a k=k^{\prime}\right\}$. Note that $k^{\prime} k^{-1}$ is the null set except when $x(k)=$ $x\left(k^{\prime}\right)$. Thus

$$
\mu\left(k^{\prime \prime} k^{-1}\right)=\sum_{k^{\prime}} \mu\left(k^{\prime \prime} k^{-1} k^{\prime-1}\right) \mu\left(k^{\prime}\right) .
$$

But $k^{\prime \prime} k^{-1}=\left\{a \mid a k=k^{\prime \prime}\right\}, k^{\prime \prime} k^{-1} k^{\prime-1}=\left\{b \mid b k^{\prime} k=k^{\prime \prime}\right\}=k^{\prime \prime} k^{\prime \prime \prime-1}$ where $k^{\prime \prime \prime}=k^{\prime} k$, so that

$$
\mu\left(k^{\prime \prime} k^{-1}\right)=\sum_{k^{\prime}} \mu\left(k^{\prime \prime} k^{-1}\right) \mu\left(k^{\prime} k^{-1}\right) .
$$

We can then regard $\mu\left(k k^{\prime-1}\right)=p\left(k \mid k^{\prime}\right)$ as the conditional probability of going from $k^{\prime}$ to $k$ for a Markov chain with state space the states $k$. The measure $\mu$ assigns positive measure to each point $k$. Notice that there is an irreducible class of states for the Markov chain with transition probabilities $\mu\left(k k^{\prime-1}\right)$ corresponding to each distinct $x$ value. By the classical results on Markov chains

This tells us that

$$
p\left(k \mid k^{\prime}\right)=\left\{\begin{aligned}
p(k) & \text { if } x\left(k^{\prime}\right)=x(k) \\
0 & \text { otherwise. }
\end{aligned}\right.
$$

$$
\mu\left(k^{\prime \prime} k^{\prime-1}\right)=\mu\left(\bigcup_{x}\left(t^{\prime \prime} t^{-1} \varphi\left(x, y^{\prime}\right)^{-1}, x, y^{\prime \prime}\right)\right)
$$

is independent of $t^{\prime}, y^{\prime}$ if $x\left(k^{\prime \prime}\right)=x\left(k^{\prime}\right)$. But expression (55) is then independent of $t^{\prime \prime}, t^{\prime}, y^{\prime}$. Then

$$
\mu\left(k^{\prime}\right)=\sum_{x\left(k^{\prime}\right)=x(k)} \mu\left(k^{\prime} k^{-1}\right) \mu(k)=\sum_{x(k)=x^{\prime}} a\left(x, y^{\prime}\right) \mu(s)=b\left(x^{\prime}, y^{\prime}\right) .
$$


Thus

$$
\mu\left(\left(\cdot, x^{\prime}, y^{\prime}\right)\right)=\sum_{x^{\prime}, t, y} \mu\left(\left(\cdot, x^{\prime \prime}, y^{\prime}\right)\right) \mu\left(\left(t, x^{\prime}, y\right)\right)=a\left(x^{\prime}\right) b\left(y^{\prime}\right)
$$

and

$$
\mu((t, x, y))=\frac{1}{n} a(x) b(y)
$$

where $n$ is the number of elements in the finite group $T$.

Notice that if $K$ contains exactly one minimal left (right) ideal, the idempotent measure $\mu$ is an $r^{*}\left(l^{*}\right)$ invariant measure in the sense of Rosen [6].

A Special Class of Semigroups. In certain contexts it is of especial interest to determine whether the semigroup $S$ is such that all elements of the kernel $K$ are left (right) annihilators, that is, $k S=k(S k=k)$ for every $k \varepsilon K$. This is of particular interest in certain structure problems for stationary processes (see [7]). We shall show that in this case not only do the averaged convolutions converge, but also the convolution sequence itself.

Theorem 7. Let $S$ be a compact Hausdorff semigroup with $k S=k(S k=k)$ for every $k \varepsilon K$, the kernel of $S$. If the semigroup generated by the spectrum of the regular measure $\nu$ on $S$ is $S$,

$$
\lim _{n \rightarrow \infty} \nu^{(n)}=\mu
$$

exists with $\mu$ having $K$ as its spectrum.

Suppose $k S=k$ for all $k \varepsilon K$. Let

$$
\mu=\lim _{n \rightarrow \infty} \frac{1}{n} \sum_{i=1}^{n} \nu^{(i)} .
$$

We shall show that $\mu=\lim _{n} \nu^{(n)}$. Let $N(k)$ be a neighborhood of $k \varepsilon K$. For sufficiently large $n \nu^{(n)}(N(k))>0$. Let $K_{N}=\left\{k^{\prime} \varepsilon K \mid k^{\prime} \varepsilon N(k)\right\}$. Take $C \subset K_{N}$ a closed set of points such that $\mu(C)>\mu\left(K_{N}\right)-\epsilon, \epsilon>0$. Given a point $k^{\prime} \varepsilon C$, there is a neighborhood $N\left(k^{\prime}\right)$ of $k^{\prime}$ such that $N\left(k^{\prime}\right) S \subset N(k)$. For, given each point $s \varepsilon S$, there are neighborhoods $N(s)$ of $s$ and $N_{s}\left(k^{\prime}\right)$ of $k^{\prime}$ such that $N_{s}\left(k^{\prime}\right)$ $N(s) \subset N(k)$. Since $S$ is compact there are a finite number of points $s_{i}$ such that $N\left(s_{i}\right)$ cover $S$. Let $N\left(k^{\prime}\right)=\bigcap N_{s i}\left(k^{\prime}\right)$. It is then clear that $N\left(k^{\prime}\right) S \subset N(k)$. Now $\bigcup_{k^{\prime} \varepsilon c} N\left(k^{\prime}\right) S \subset N(k)$. Let $N=\bigcup_{k^{\prime} \varepsilon c} N\left(k^{\prime}\right)$. Then for some sufficiently large $n, \nu^{(n)}(N)>\mu\left(K_{N}\right)-\epsilon$. But then for all $m>n$

$$
\nu^{(m)}(N(k))=\int \nu^{(n)}\left(N(k) s^{-1}\right) \nu^{(m-n)}(d s) \geqq \nu^{(n)}(N)>\mu\left(K_{N}\right)-\epsilon .
$$

Thus

$$
\varliminf_{m \rightarrow \infty} \nu^{(m)}(N(k)) \geqq \mu(N(k)) .
$$


This is not possible unless

$$
\lim _{m \rightarrow \infty} \nu^{(m)}(N(k))=\mu(N(k)) .
$$

Thus $\lim \nu^{(m)}=\mu$. The proof is essentially the same when $S k=k$ for every $k \varepsilon K$.

A very simple but interesting application of Theorem 7 can be given. Let $\nu$ be a regular measure on the $n \times n$ probability matrices, that is, matrices with nonnegative elements and row sums one. Suppose the spectrum of $\nu$ contains a matrix with identical rows. The assumption of Theorem 6 is then satisfied by the semigroup of matrices $S$ generated by the spectrum of $\nu$. Thus $\lim \nu^{(m)}$ exists and is concentrated on the kernel $K$ of $S$ which consists of matrices with identical rows. Let $a_{1}, \cdots, a_{n}, \sum a_{i}=1$, be the random elements of the common row for the limit measure concentrated on the matrices of $K$. Then the characteristic function

$$
\begin{aligned}
& \varphi\left(z_{1}, \cdots, z_{n-1}\right)=\mathrm{E} \exp \left\{i \sum_{j=1}^{n-1} z_{j} a_{j}\right\} \\
&= \mathrm{E} \exp \left\{i \sum_{1}^{n-1} z_{i}\left(\sum_{k=1}^{n-1} a_{k} p_{k j}+\left[1-\sum_{1}^{n-1} a_{k}\right] p_{n i}\right)\right\} \\
&= \mathrm{E}\left[\mathrm{E}\left(\exp \left\{i \sum_{1}^{n-1} z_{i} p_{n j}\right\} \exp \left\{i \sum_{1}^{n-1} z_{i} \sum_{1}^{n-1}\left(p_{k i}-p_{n i}\right) \mid p_{k i}\right\}\right]\right. \\
&= \int \varphi\left(\sum_{1}^{n-1} z_{j}\left(p_{1 i}-p_{n j}\right), \cdots, \sum_{1}^{n-1} z_{j}\left(p_{n-1, i}-p_{n j}\right)\right) \\
& \cdot \exp \left\{i \sum_{1}^{n-1} z_{j} p_{n j}\right\} d \nu(p) .
\end{aligned}
$$

\section{BiBLIOGRAPHY}

[1] R. Beldman, Limit theorems for noncommutative operations I, Duke Math. Journ. 21, (1954).

[2] N. Dunford \& J. T. Schwartz, Linear Operators, Part I, 1958 (New York).

[3] U. Grenander, Some nonlinear problems in probability theory, H. Cramer Volume (1959).

[4] Y. KaWAdA \& K. ITo, On the probability distribution on a compact group I, Proc. Phys.Math. Soc. of Japan, 22 (1940), pp. 977-998.

[5] K. Numakura, On bicompact semigroups, Math. Journ. of Okayama Univ. (1952), pp. 99-108.

[6] W. G. Rosen, On invariant means over compact semigroups, Proc. Amer. Math. Soc., 7 (1956), pp. 1076-1082.

[7] M. RosenblatT, Stationary processes as translates of functions of independent random variables, Journ. Math. and Mech., 8 (1959), pp. 665-682.

[8] A. D. WALLACE, The Rees-Suschkewitsch structure theorem for compact simple semigroups, Proc. Nat. Acad. Sci. U.S.A., 42 (1956), pp. 430-432.

Indiana University

Bloomington, Indiana 